\title{
Detecting Errors in Cognitive Models Using Visualization Metaphors of Fuzzy Cognitive Maps *
}

\author{
Ruslan Isaev ${ }^{[0000-0003-3263-4051]}$ and Aleksandr Podvesovskii [0000-0002-1118-3266] \\ Bryansk State Technical University, Bryansk, Russia \\ ruslan-isaev-32@yandex.ru, apodv@tu-bryansk.ru
}

\begin{abstract}
Verification of cognitive models is one of the most important stages in their construction, since reliability of results of subsequent modeling largely depends on the successful implementation of verification. The paper considers the problem of verifying cause-and-effect relationships in cognitive models based on the use of fuzzy cognitive maps. It is noted that increasing the effectiveness of cognitive model verification is possible by activating analyst's cognitive potential. The most natural way of such activation is to increase cognitive clarity of the model through the use of visualization capabilities. For this purpose, a number of metaphors for visualizing fuzzy cognitive maps have been proposed, aimed at increasing their cognitive clarity during verification. Each of the metaphors is focused on the visualization of a certain type of fragments of a fuzzy cognitive map potentially containing errors, redundancy or incompleteness and therefore of interest from the point of view of verification. The first considered visualization metaphor is intended to display the cycles that are part of a cognitive graph. The second metaphor focuses on the mapping of transitive paths between concepts. Finally, the third metaphor is aimed at eliminating cognitive model incompleteness, which consists in the lack of relationships between some concepts. Examples are given of applying the proposed visualization metaphors to increase cognitive clarity of the visual image of the verified fuzzy cognitive map.
\end{abstract}

Keywords: Cognitive Modeling, Fuzzy Cognitive Map, Visualization Metaphor, Cognitive Clarity, Verification.

\section{Introduction}

This paper continues a series of publications of the authors' research materials in the field of visualization of cognitive models based on fuzzy cognitive maps (FCM). An FCM reflects a researcher's subjective idea of a system in the form of a set of semantic categories (called factors or concepts) and a set of causal relationships between them [1]. Thus, an FCM can be visualized in the form of a weighted directed graph the

Copyright (C) 2020 for this paper by its authors. Use permitted under Creative Commons License Attribution 4.0 International (CC BY 4.0).

* The reported study was funded by RFBR, project number 19-07-00844. 
vertices of which correspond to concepts and the edges - to cause-and-effect relationships.

One of the conditions for effective work with a cognitive model is to ensure its visual representation. In [2, 3], the authors proposed an approach to FCM visualization based on the visualization metaphor concept. Visualization metaphor traditionally includes two components: spatial metaphor and representation metaphor [4].

The spatial metaphor defines general principles of transferring a visualized object into the visual model space. With regard to an FCM, such a metaphor is based on graph visualization algorithms and formalized criteria of cognitive clarity [3]. These criteria describe requirements for the FCM visual image quality. Observing these requirements simplifies visual perception of the cognitive model by the analyst. This leads to a general increase in the speed of working with the model, and also helps to reduce the number of errors made at various stages of modeling.

The representation metaphor used below is responsible for finalizing the resulting visual image in order to identify its components that are most important in the context of the problem being solved. A number of different representation metaphors are used in the visualization of the FCM taking into account the analyst's needs at different stages of cognitive modeling.

The paper investigates capabilities of FCM visualization when solving one of the important tasks of cognitive modeling - namely, cognitive model verification. The research is based on the hypothesis of an increase in the efficiency of FCM verification by increasing cognitive clarity of its visual image.

\section{The task of verifying cognitive models}

The task of verifying a cognitive model is an important task solved at the stage of model building and is aimed at identifying a possible discrepancy between the model and the modeled system itself. This discrepancy can be expressed in the following basic forms.

1. The cognitive model may lack concepts that reflect important parameters of the modeled system, or, conversely, there may be redundant concepts that are not important in relation to the modeling goal.

2. A set of cause-and-effect relationships given on a set of concepts can be characterized by both incompleteness and redundancy.

3. Errors can be made when setting parameters of cause-and-effect relationships (direction, sign, intensity).

Search techniques and eliminating inconsistencies of the first type require highly qualified experts and deep understanding of the subject area and, as a rule, are the most difficult to formalize. One of the possible approaches here may be ontological engineering [5].

Errors made in the parametric identification of the model (the third type of discrepancy) are the least obvious for detection and most often can be detected directly from the results of modeling, based on the analysis of their plausibility [6]. At the same time, certain reliability control of parameters of FCM relationships can be performed within the framework of the identification methods themselves [7]. 
The proposed research focuses on the second type of discrepancy as the easiest one to apply formal verification methods. In addition, as will be shown below, in this case it becomes possible to effectively combine formal methods with methods based on the activation of the analyst's cognitive capabilities.

It should be noted here that when identifying the redundancy of a set of relationships, two types of elements of cognitive graph structure are most important for analysis: oriented cycles and pairs of transitive paths. The importance of oriented cycles stems from the fact that they, representing feedback loops, in some cases can lead to a violation of cognitive model stability in the course of its scenario analysis. Pairs of transitive paths describe alternative mechanisms of concept interaction which must be assessed by the analyst, on the one hand, for mutual consistency, and on the other hand, for the need to be reflected in the model in general.

The problem of search for oriented cycles and pairs of transitive paths in an FCM belongs to common problems in graph theory. Therefore, further we describe how to process and present the results of such a search using visualization capabilities.

\section{Application of visualization metaphors of fuzzy cognitive maps in the process of cognitive model verification}

Let us consider some FCM visualization metaphors that can be used in the process of verifying cognitive models. The use of these and other similar metaphors increases cognitive clarity of verified models, which will help to activate the analyst's cognitive abilities when solving a number of specific tasks during verification. To illustrate the results of applying the proposed metaphors, we will use the FCM shown in Fig. 1.

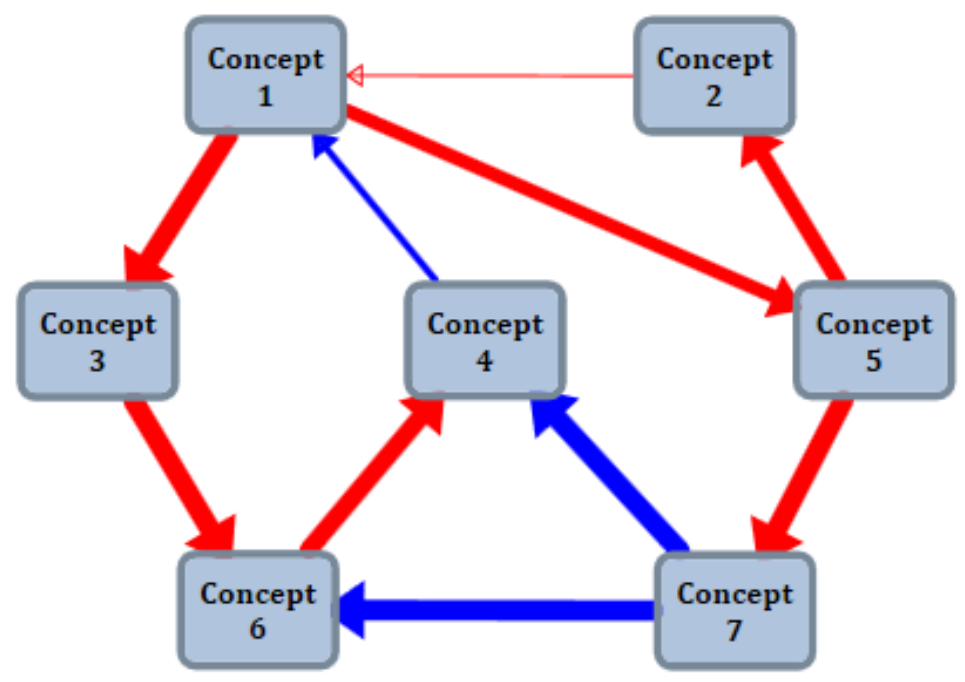

Fig. 1. Example of an FCM subject to verification 


\subsection{Visualization of oriented cycles}

Let us consider a visualization metaphor designed to display oriented cycles found within an FCM. Suppose one of the cycles is set for the visualization. The essence of the spatial component of this metaphor consists in depicting the FCM in such a way that the criterion of unidirectionality of successive edges is maximized on the set of edges included in a given cycle. Less formally, the metaphor seeks to place the vertices of the cognitive graph in such a way as to provide a unidirectional image of as many edges within the cycle as possible. It has been noted [8] that such placement contributes to the cycle coverage "at a glance". In this case, it is advisable to choose the direction "left-to-right" or "top-down" as the priority direction (that is, the direction of most edges). This is due to the criterion of optimizing edge directions which is also taken into account [3].

The corresponding representation metaphor is characterized by the concentration of the analyst's attention directly on the cycle under consideration. A simple solution to this problem could be complete absence of images of "excess" sections of the FCM. However, this approach has an obvious disadvantage of removing the context useful for verification from the analyst's perception. Therefore, it seems more rational to depict all the FCM elements that are not included in the cycle semi-transparent. It should also be noted that it is advisable to individually adjust the degree of transparency taking into account peculiarities of a particular analyst's perception.

Fig. 2 shows an example of application of this metaphor to the test FCM when visualizing the cycle "1-5-7-6-4-1". It is easy to see that restructuring the FCM image is much better (compared to the original metaphor) in attracting the analyst's attention to the selected cycle. This allows us to speak of an increase in cognitive clarity of the model in the context of the problem under consideration.

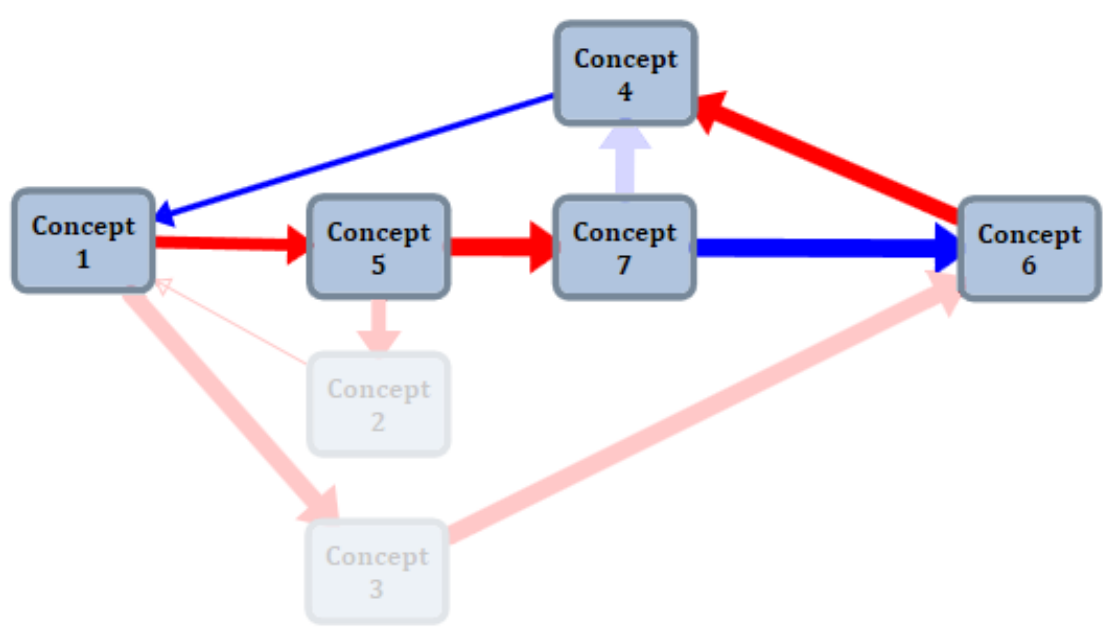

Fig. 2. Example of applying the cycle visualization metaphor 
Let us note that if an FCM includes several cycles, it becomes necessary to specify the order of their presentation to the analyst. At the same time, it is advisable to take into account that the risk of FCM stability disruption is primarily due to the presence of cycles with a positive weight (the weight of a cycle refers to the product of the weights of influences included in it): concepts in such a cycle tend to intensify their own changes. Therefore, if there are several such cycles, priority should be given to the cycles with the highest weight.

\subsection{Visualization of pairs of transitive paths}

The next visualization metaphor is intended to depict pairs of transitive paths between concepts. As in the previous case, the spatial component of this metaphor takes into account the criterion of the unidirectionality of successive edges, but additionally maximizes the symmetry of the subgraph subject to visualization [3]. By analogy with the previous case, the representation metaphor uses the effect of a semi-transparent image of "excess" graph sections to focus the analyst's attention on the selected transitive paths.

Fig. 3 shows an example of this metaphor application when visualizing a pair of paths "1-3-6-4" and "1-5-7-4". Due to equal path lengths, it was possible to ensure the symmetry of the target subgraph about the horizontal axis.

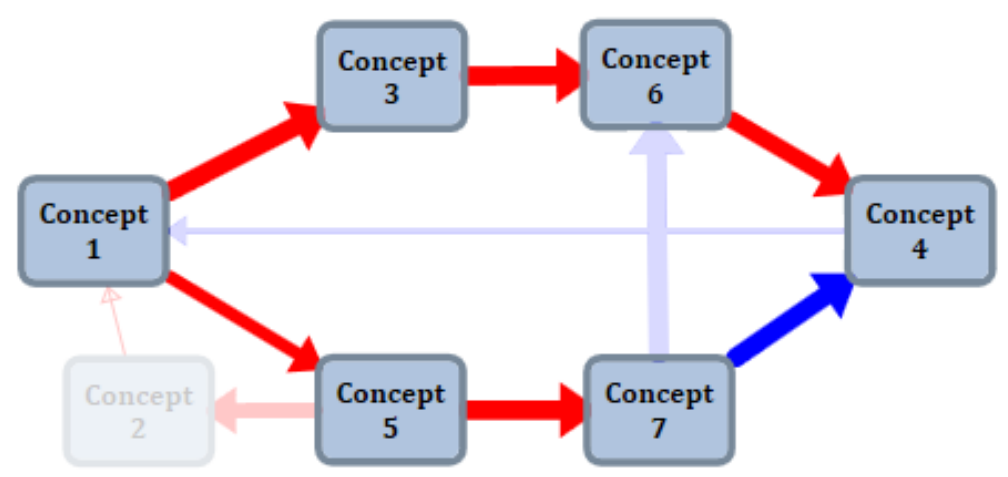

Fig. 3. Example of metaphor application for visualizing pairs of transitive paths

By analogy with the previous metaphor, the priority direction of the edges can be either "left-to-right" or "top-down", depending on the analyst's preferences.

\subsection{Visualization of a missing relationship between concepts}

If the two previous visualization metaphors were aimed at eliminating the redundancy of a cognitive model, then this metaphor, on the contrary, is aimed at eliminating the incompleteness of the model. In this case, incompleteness means lack of relationships between some concepts. 
It should be noted here that absence of oriented paths between some pairs of concepts in a cognitive graph is a typical situation when building a cognitive model. As a consequence, even in the long term, changes in the states of some concepts will not affect the states of a number of other concepts. From the point of view of object interpretation, this means cause-and-effect independence of the corresponding parameters of the modeled system from each other; this is quite admissible. Nevertheless, in a number of cases, a missing relationship occurs by mistake during the FCM construction, and such situations require detection and correction.

Pairs of concepts which lack oriented paths between them can be easily identified based on the operation of transitive closure of a cognitive matrix corresponding to the FCM under study. The sign of the absence of a path between the concepts is the equality to zero of the corresponding element of the transitively closed matrix.

In general, there are many unrelated pairs of concepts in an FCM. Thus, in addition to detecting such pairs, it is also necessary to determine the order of their presentation to the analyst for the purpose of determining whether it is expedient to add a relationship. It is advisable to give the highest priority to such pairs of concepts the addition of a relationship between which will have the most significant impact on the modeling results.

Let the FCM in Fig. 1 initially lack relationship directed from Concept 6 to Concept 4 . It is easy to see that this leads to the absence of oriented paths from Concept 3 to all concepts except Concept 6 , as well as from Concept 6 to all concepts.

Let us suppose that a relationship is added from Concept 6 to any of the concepts numbered 1, 2, 4, 5. Obviously, this will lead to the emergence of oriented paths from Concept 6 itself to all concepts, as well as from Concept 3 to all concepts. If such a relationship is added from Concept 3 , then Concept 6 will remain isolated. Therefore, it is advisable to assign a higher priority to considering Concept 6 as a concept-cause.

Further, it is required to determine the order of presentation of potential conceptconsequences, that is, concepts with numbers 1, 2, 4, 5. A possible solution here may be to focus on the intensity of influences exerted by these concepts on the other FCM concepts. This information can also be obtained from a transitively closed matrix. The greatest total influence on the concepts within the FCM is exerted by Concept 1 .

An example of using a visualization metaphor taking into account the above reasoning is shown in Fig. 4. The analyst is invited to add a relationship from Concept 6 to Concept 1 , and he can either agree with this proposal or refuse it. If the analyst agrees to add a relationship, then he needs to set its parameters, which, in turn, requires the use of methods of FCM parametric identification [7].

An important feature of the proposed visualization metaphor is the possibility of adjusting its spatial component in order to increase cognitive clarity of the visual image of the FCM. So, if the concepts presented to the analyst in order to add a relationship are situated far from each other and are separated by other elements of the FCM, then visual image rebuilding is performed, aimed simultaneously at the spatial convergence of these concepts and at maintaining the usual location of the remaining FCM elements. Fig. 5 exemplifies how the metaphor works in such a situation. It should be noted that from the point of view of automating visual image correction, an approach based on the simulated annealing method proposed in [9] is of interest. 
Detecting Errors in Cognitive Models Using Visualization Metaphors of Fuzzy ... 7

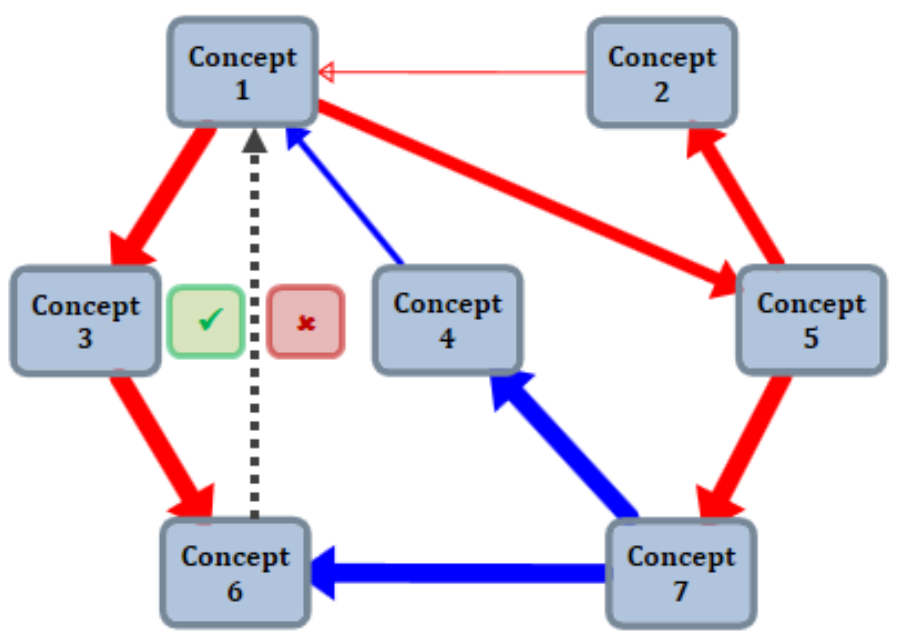

Fig. 4. Example of using a visualization metaphor to eliminate a missing relationship between concepts (the case of preserving the original spatial metaphor)

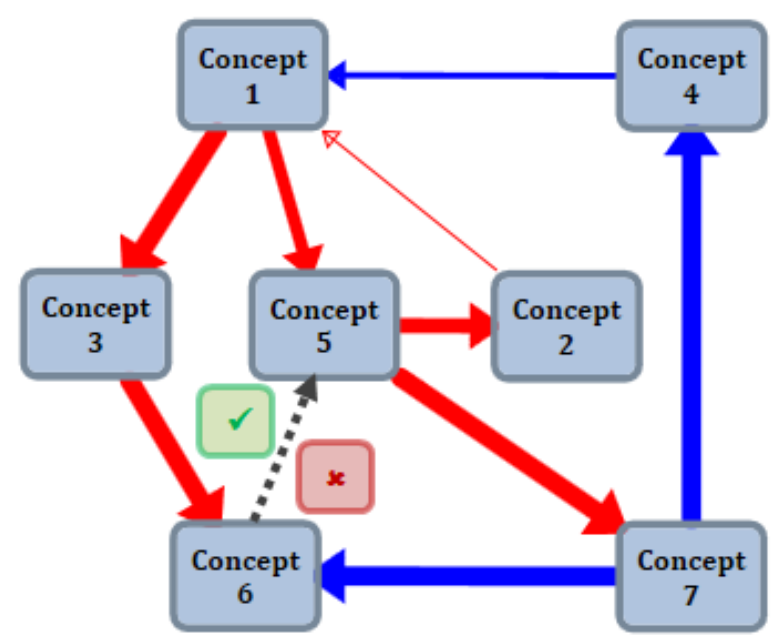

Fig. 5. Example of using a visualization metaphor to eliminate a missing relationship between concepts (the case of spatial metaphor correction)

\section{Conclusion}

The paper presents possibilities of applying the approach to FCM visualization based on visualization metaphors for verification of fuzzy cognitive models. Examples of visualization of situations that may characterize the incompleteness or redundancy of a set of cause-and-effect relationships between concepts are considered. It is shown that the effectiveness of cognitive model verification can be increased by increasing cognitive clarity of the visual image of the FCM underlying it. 
Areas for further research include:

- Development and research of other visualization metaphors useful in FCM verification.

- Development of a general verification methodology that allows combining formal mathematical methods with subsequent visual processing of the results obtained on the basis of visualization metaphors.

- Software implementation of the developed methodology in the form of a cognitive model verification subsystem as part of IGLA DSS [10], as well as its performance evaluation in the construction and study of fuzzy cognitive models of real applied problems.

\section{References}

1. Borisov, V.V., Kruglov, V.V., Fedulov, A.S.: Nechetkie modeli i seti [Fuzzy Models and Networks]. Goryachaya Liniya - Telekom, Moscow, Russia (2012). [in Russian]

2. Podvesovskii, A.G., Isaev, R.A.: Visualization Metaphors for Fuzzy Cognitive Maps. Scientific Visualization 10(4), 13-29 (2018). doi: 10.26583/sv.10.4.02

3. Podvesovskii, A.G., Isaev, R.A.: Constructing Optimal Visualization Metaphor of Fuzzy Cognitive Maps on the Basis of Formalized Cognitive Clarity Criteria. Scientific Visualization 11(4), 115-129 (2019). doi: 10.26583/sv.11.4.10

4. Zakharova, A.A., Shklyar, A.V.: Visualization Metaphors. Scientific Visualization 5(2), 1624 (2013).

5. Tiuriumin, V., Massel A.: Integration of Situation Semantic Models Based on Ontology System. In: Proceedings of 3rd Russian-Pacific Conference on Computer Technology and Applications (RPC). pp. 1-5. IEEE, Vladivostok, Russia (2018). doi: 10.1109/RPC.2018.8482232

6. Kulinich, A.A.: Verifikatsija kognitivnyh kart na osnove ob'jasnenija prognozov [Cognitive Maps Verification Based on Processes Explanation]. Upravlenie bol'shimi sistemami [Large-Scale Systems Control] 30.1, 453-469 (2010). [in Russian].

7. Podvesovskii, A.G., Isaev, R.A.: Identification of Structure and Parameters of Fuzzy Cognitive Models: Expert and Statistical Methods. International Journal of Open Information Technologies 7(6), 35-61 (2019).

8. Abramova, N.A., Voronina, T.A., Portsev, R.Y. O metodah podderzhki postroeniya i verifikacii kognitivnyh kart s primeneniem idej kognitivnoj grafiki [Ideas of Cognitive Graphics to Support Verification of Cognitive Maps]. Upravlenie bol'shimi sistemami [Large-Scale Systems Control] 30.1, 411-430 (2010). [in Russian].

9. Lee, Y.-Y., Lin, C.-C., Yen, H.-C.: Mental Map Preserving Graph Drawing Using Simulated Annealing. Information Sciences 181(19), 4253-4272 (2011). doi: 10.1016/j.ins.2011.06.005

10. Zakharova, A.A., Podvesovskii, A.G., Isaev, R.A. Matematicheskoe i programmnoe obespechenie podderzhki kognitivnogo modelirovanija slabostrukturirovannyh organizacionno-tehnicheskih system [Mathematical and Software Support for Cognitive Modeling of Semi-structured Organizational and Technical Systems]. In: CPT2019 International conference Proceedings, pp. 131-141. Pub. NNGASU and SRCIPT, Nizhniy Novgorod (2019). [in Russian]. 